\title{
Euclidean geometry problems rephrased in terms of midpoints and point-reflections
}

\author{
Victor Pambuccian \\ Victor Pambuccian studierte von 1978 bis 1982 Mathematik an der Universität Buka- \\ rest und promovierte 1993 an der University of Michigan in Ann Arbor. Seit 1994 ist \\ er Professor an der Arizona State University West. Sein Hauptforschungsgebiet ist die \\ axiomatische Grundlegung der Geometrie.
}

When we conjecture a theorem in geometry, we first try to prove it using whatever means we find at our disposal. After establishing its truth, we may follow it up by a reverse analysis, first described by Pappus of Alexandria as analysis, asking not whether the theorem in question holds, but what does one need to assume for it to hold (see [10] for the history of the regressive undertaking). Hilbert [4] expressed this enterprise with his characteristic eloquence:

Unter der axiomatischen Erforschung einer mathematischen Wahrheit verstehe ich eine Untersuchung, welche nicht dahin zielt, im Zusammenhange mit jener Wahrheit neue oder allgemeinere Sätze zu entdecken, sondern die vielmehr die Stellung jenes Satzes innerhalb des Systems der bekannten Wahrheiten und ihren logischen Zusammenhang in der Weise klarzulegen sucht, daß sich sicher angeben läßt, welche Voraussetzungen zur Begründung jener Wahrheit notwendig und hinreichend sind.

Im Zusammenhang mit dem Finden und Beweisen mathematischer Sätze ist auch die Frage nach einem minimalen Axiomensystem, mit Hilfe dessen die entsprechenden Sätze hergeleitet werden können, ein zentrales Element mathematischen Arbeitens. In dem nachfolgenden Artikel nimmt sich der Autor dieser Frage im Hinblick auf vier Sätze der Elementargeometrie an, darunter der Satz, dass Schwerpunkt, Umkreismittelpunkt und Höhenschnittpunkt auf einer Geraden, der sogenannten Eulerschen Geraden, liegen. In der Regel werden diese vier Sätze mit Hilfe des Axiomensystems, das der Euklidischen Geometrie zugrunde liegt, bewiesen. Der Autor zeigt nun, dass sie bereits mit Hilfe dreier einfacher Axiome, die minimale Forderungen an die Punktspiegelungsoperation stellen, beweisbar sind. 
A reverse analysis is admittedly less well defined than the proof of a statement inside an axiom system, as it is not at all clear what should count for an "assumption", nor which set of assumptions ought to be declared optimal.

What we search in a reverse analysis is to minimize the number and the complexity of both the notions and the assumptions used in the proof of a specific statement, and we may well arrive at several different axiom systems, expressed with different primitive notions, which are not at all comparable. The reverse analyses which were carried out for two geometric problems in [8] and [9] arrived at precisely such different and incomparable axiom systems.

In the present paper we present four elementary geometry problems, one of which is the Euler line problem, whose reverse analysis leads to the same particularly rudimentary axiom system, for a very weak geometry with a Euclidean flavour.

It has been noticed in [1] and [11] that the Euler line can be generalized to an affine setting. It turns out that much less than affine geometry is enough to prove a generalization of the Euler line statement.

All the results we have in mind can be formulated in terms of point-reflections and midpoints alone. The basic axioms describing the Euclidean behaviour of these two binary operations were proposed by D. Vakarelov [13]. The axiom system is stated in a language with one sort of variables, to be interpreted as points and to be denoted by uppercase Latin characters, in terms of a single binary operation $\cdot$, with $A \cdot B$ to be read as the reflection of $A$ in $B$. Its axioms are

A $1 \quad A \cdot A=A$

A $2(\forall A)(\forall B)\left(\exists^{=1} X\right) B \cdot X=A$

A $3((A \cdot B) \cdot C) \cdot D=((A \cdot D) \cdot C) \cdot B$

The axiom A2 states the existence and uniqueness of the midpoint of any pair of points $A$ and $B$, which will be denoted by $\mu(A B)$, whereas $\mathrm{A} 3$ is a closure condition - one could say "configuration theorem" (Schnittpunktsatz), although we do not have actual "lines" in our "geometry" - expressed in the language of point reflections.

Following Vakarelov, we will introduce a few definitions that will bring us closer to the concepts of affine geometry. Let $\mathfrak{M}$ be a model of the above axioms. For points $A, B, C, D$ in $\mathfrak{M}$, we say that the pair $(A, B)$ is equivalent to the pair $(C, D)$, and write $(A, B) \sim$ $(C, D)$, if there exists a point $X$ such that $A \cdot X=D$ and $B \cdot X=C$. The relation $\sim$ is an equivalence relation, and an addition can be defined on the set $\mathcal{S}:=\mathfrak{M} / \sim$ of equivalence classes, which turns $\mathcal{S}$ into an abelian group in which, for all $a$, the equation $x+x=a$ has a unique solution $x$. Fixing a point $O$ in $\mathfrak{M}$, and writing $[A, B]$ for the equivalence class under $\sim$ of $(A, B)$, we obtain that $[O, A \cdot B]=2[O, B]-[O, A]$, and $[O, \mu(A B)]=\frac{1}{2}([O, A]+[O, B])$. To simplify the notation, and bring it in line with the one more familiar from the complex plane (see e.g. [6]), we may think of $[O, A]$ as the affix of point $A$, and write instead of $[O, A]$ simply $A$. Our formulas for the operations of 
reflection in a point and midpoint thus become those of Grassmann [2]:

$$
A \cdot B=2 B-A \quad \text { and } \quad \mu(A B)=\frac{A+B}{2} .
$$

We can also define a collinearity relation $\operatorname{Col}$, with $\operatorname{Col}(A, B, C)$ to be read as "points $A$, $B, C$ are collinear (but not necessarily different)", as the smallest relation satisfying, for all $A, B, C, D$, the following conditions: $\operatorname{Col}(A, B, \mu(A B)) ; \operatorname{Col}(A, B, A)$; if $\operatorname{Col}(A, B, C)$ then $\operatorname{Col}(C, B, A)$ and $\operatorname{Col}(B, A, C)$; and if $A \neq B, \operatorname{Col}(A, B, C), \operatorname{Col}(A, B, D)$, then $\operatorname{Col}(A, C, D)$. With its help we can also define a notion of parallelism of segments $\|$, by stipulating that the segment $A B$ be parallel to the segment $C D$, i.e., $A B \| C D$, if and only if $A \neq B, C \neq D$, and there exist $E$ and $F$, with $E \neq F$, both collinear with $C$ and $D$, such that $(A, B) \sim(E, F)$, or else $A=B$ and $C=D$. Given (1), we have $A B \| C D$ if and only if the affixes satisfy $B-A=\lambda(D-C)$, where $\lambda$ is a rational number of the form $\frac{m}{2^{n}}$, where $m \in \mathbb{Z} \backslash\{0\}$ and $n \in \mathbb{N} \cup\{0\}$. The particular case of the parallelism relation $(A, B) \sim(C, D)$ is equivalent with $B-A=D-C$.

We will now show that four problems usually stated within Euclidean geometry, involving metric notions (such as orthogonality and segment-congruence), can be rephrased as statements in terms of point-reflections only and can be proved inside the axiom system A1-A3.

Not only can they be thus rephrased, but in some sense, this is all they require, and any proof carried out in the Euclidean framework will have to be unnecessarily complex. In our setting the proofs turn out to be immediate consequences of the associativity and commutativity of addition.

1. The center of mass $G$, the circumcenter $O$, and the orthocenter $H$ of a triangle are collinear, and $O G: G H=1: 2$.

2. The four lines, each drawn from the midpoint of a side of a cyclic quadrilateral perpendicular to the opposite side, are concurrent.

3. Given a triangle $A B C$, let $A^{\prime}, B^{\prime}, C^{\prime}$ be the reflections of the circumcenter $O$ in $\mu(B C), \mu(C A), \mu(A B)$. Prove that the midpoints of $A A^{\prime}, B B^{\prime}$, and $C C^{\prime}$ coincide.

4. In a triangle $A B C$, the midpoint $M$ of side $B C$, the reflection of $A$ in the circumcenter $O$, and the orthocenter $H$ are collinear.

The first is the Euler line theorem, the second can be found in [5, p. 36], and also showed up in the first round of the 1977 edition of the Bundeswettbewerb Mathematik, the third is Problem 11003 in [3], and the fourth is Problem 232 in [12].

Their rephrasing as statements in the language of point-reflections reads as follows:

1'. Given points $O, A, B, C, G, M=\mu(A B), N=\mu(A C), P=\mu(B C)$, and $Q=$ $O \cdot G$, such that $G \cdot(P \cdot G)=A$, there is a point $X$ such that $A X\|O P, B X\| O N$, and $C X \| O M$. The point $X$ is precisely $G \cdot Q$. 


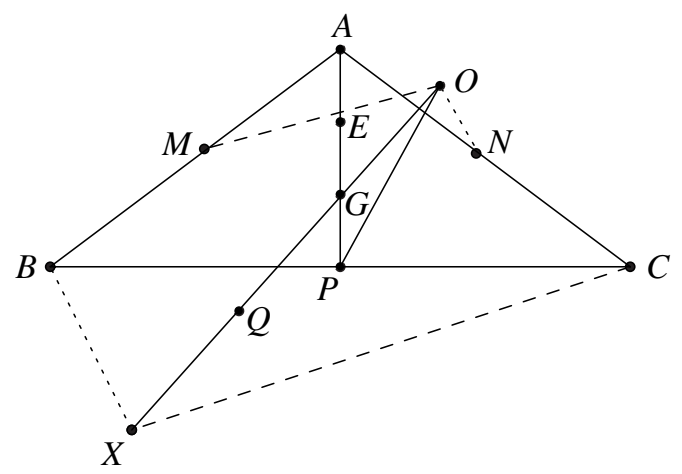

Fig. 1: The statement $\mathbf{1}^{\prime}$

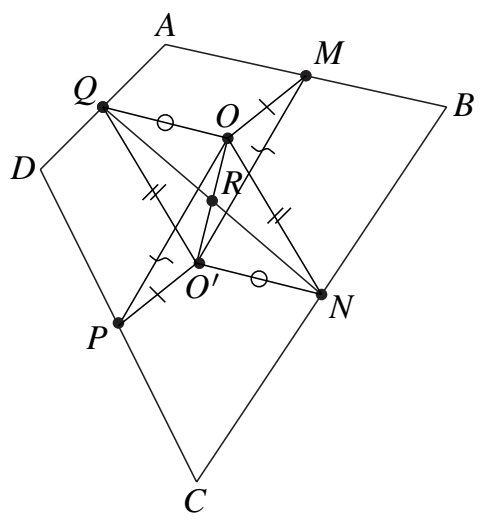

Fig. 2: The statement $\mathbf{2}^{\prime}$

$\mathbf{2}^{\prime}$. Let $A, B, C, D, O$ be five points and let $M=\mu(A B), N=\mu(B C), P=\mu(C D)$, $Q=\mu(D A), O^{\prime}=O \cdot \mu(Q N)$. Then $(O, M) \sim\left(P, O^{\prime}\right),(O, N) \sim\left(Q, O^{\prime}\right)$, $(O, P) \sim\left(M, O^{\prime}\right),(O, Q) \sim\left(N, O^{\prime}\right)$ (put differently, the four parallels from the midpoints of each side to the lines joining $O$ to the midpoint of the "opposite" side meet in $O^{\prime}$ ).

$3^{\prime}$. Let $A, B, C, O$ be four points, and let $A^{\prime}=O \cdot \mu(B C), B^{\prime}=O \cdot \mu(A C), C^{\prime}=$ $O \cdot \mu(A B)$. Then $\mu\left(A A^{\prime}\right)=\mu\left(B B^{\prime}\right)=\mu\left(C C^{\prime}\right)$.

$4^{\prime}$. Let $A, B, C, O$ be four points and let $A^{\prime}=A \cdot O, M=\mu(B C), N=\mu(B A)$, $P=\mu(A C), H=A^{\prime} \cdot M$. Then $O M\|A H, O N\| C H, O P \| B H$.

That the statements do require a Euclidean behaviour of parallels can be easily seen from the fact that these statements fail to hold in hyperbolic geometry (in fact, whenever the underlying metric is non-Euclidean, see [7] for the Euler line case). Thus Axiom A1 will surely have to hold to reflect the most basic property of symmetry in a point, and we do need to have midpoints to even formulate the statements to be proved, so A2 needs to hold. 


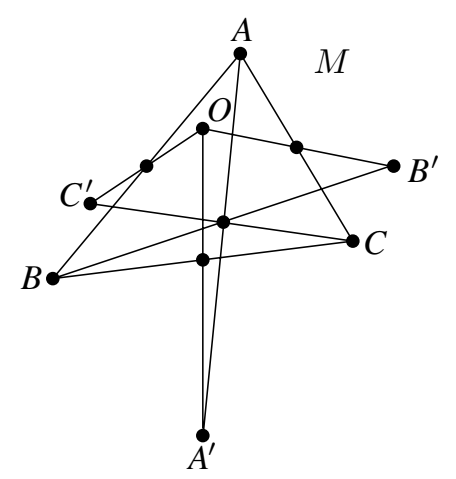

Fig. 3: The statement $\mathbf{3}^{\prime}$

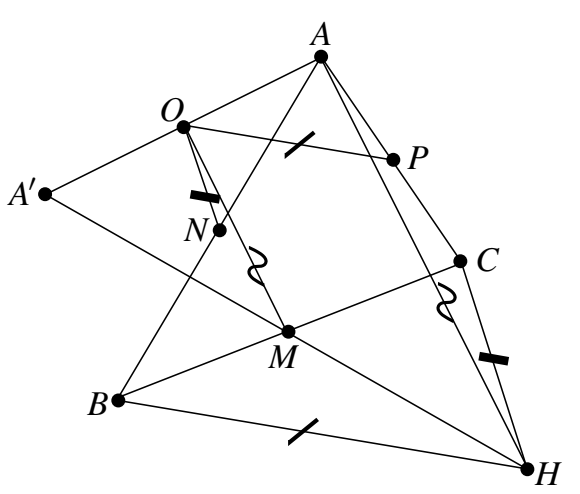

Fig. 4: The statement $\mathbf{4}^{\prime}$

Since the statements hold only when parallels behave in a Euclidean manner, A3 - or an axiom similar to it - is needed as well.

The proofs are immediate, bearing in mind (1). To simplify computations even more, we may assume that the affix $O$ is the neutral element of the abelian group $\mathcal{S}$.

In $\mathbf{1}^{\prime}$ the hypothesis $G \cdot(\mu(B C) \cdot G)=A$ leads to $A=3 G-B-C$, and, since $X=3 G$, the statement $A X \| O \mu(B C)$ holds, as it is equivalent to $X-A=3 G-A=B+C=$ $2\left(\frac{B+C}{2}-O\right)$, and the other relations follow analogously.

In $2^{\prime} O^{\prime}=\frac{A+B+C+D}{2}, M=\frac{A+B}{2}, P=\frac{C+D}{2}$, so $(O, M) \sim\left(P, O^{\prime}\right)$ is equivalent to $\frac{A+B}{2}=\frac{A+B+C+D}{2}-\frac{C+D}{2}$, and the other relations follow analogously.

In $\mathbf{3}^{\prime} A^{\prime}=B+C, B^{\prime}=C+A, C^{\prime}=A+B$, so $\mu\left(A A^{\prime}\right)=\mu\left(B B^{\prime}\right)=\mu\left(C C^{\prime}\right)=\frac{A+B+C}{2}$.

In $4^{\prime} A^{\prime}=-A, M=\frac{B+C}{2}, H=B+C+A$, so $O M \| A H$, as $M-O=\frac{B+C}{2}=\frac{1}{2}(H-A)$, and the other relations are proved analogously. 


\section{References}

[1] Boon, A.W.: Die Eulersche Gerade - Ein Beweis. Euclides (Groningen) 57 (1978), 56-57.

[2] Grassmann, H.: A new branch of mathematics. The Ausdehnungslehre of 1844 and other works. Translated from the German and with a note by Lloyd C. Kannenberg. Open Court, Chicago 1995.

[3] Gray, S.B.: Problem 11003. Amer. Math. Monthly 110 (2003), 241.

[4] Hilbert, D.: Über den Satz von der Gleichheit der Basiswinkel im gleichschenkligen Dreieck. Proc. London Math. Soc. 35 (1902/1903), 50-68.

[5] Honsberger, R.: Episodes in Nineteenth and Twentieth Century Euclidean Geometry . MAA, Washington 1995.

[6] Jeger, M.: Komplexe Zahlen in der Elementargeometrie. Elem. Math. 37 (1982), 136-147.

[7] Künneth, H.: Der Schwerpunkt, die Eulersche Gerade und der Feuerbachsche Kreis in der absoluten Geometrie. Jahresber. Deutsch. Math.-Verein. 43 (1933), 65-77.

[8] Pambuccian, V.: A methodologically pure proof of a convex geometry problem. Beiträge Algebra Geom. 42 (2001), 401-406.

[9] Pambuccian, V.: On the planarity of the equilateral, isogonal pentagon. Math. Pannon. 14 (2003), 101-112.

[10] Peckhaus, V.: Regressive Analysis. Log. Anal. Hist. Philos. 5 (2002), 97-110.

[11] Snapper, E.: An affine generalization of the Euler line. Amer. Math. Monthly 88 (1981), 196-198.

[12] Ţiţeica, G.: Recueil de problèmes de géométrie (Rumanian). Editura Tehnică, Bucharest 1962.

[13] Vakarelov, D.: Algebraic foundations of central symmetry, of rotation and of homothety (Bulgarian). Annuaire Univ. Sofia Fac. Math. Inform. 63 (1968/1969), 121-166.

Victor Pambuccian

Department of Integrative Studies (MC 3051)

Arizona State University West

Phoenix, AZ 85069-7100, USA

e-mail: pamb@math.west.asu.edu 